\title{
2005 CAEP/ACMU Scientific Abstracts Key Word Index
}

\section{KEY WORD}

A

abdominal pain 75

access

activated charcoal

acute coronary syndrome

adolescents

advanced directive

advanced life support

adverse drug effects

aeromedical

airway

airway management

alcohol withdrawal

analgesia

anion gap

anxiety

appendicitis

assessment

asthma

ATLS

BNP
burns

B

cardiac arrest

C

cellulitis

chest pain

children

clavicle fractures

clinical guidelines

clinical prediction rule

clinical scoring

clinical trials

communicable diseases

communication

computed tomography

congestive heart failure

consent

CQI

critical care

critical incidents

crowding

CTAS

cycling

D

database

ABS. NO.

113

22

102

47

1,2

$72,77,83$

95

76

51

38,115

75,107

114

98

54

110

24,25

43

$1,2,4,17,71$, $91,44,47,50,55$

39,53

22

75,80

80

$80,99,106$

$45,50,54$

78

92

37

31,46

20,70

81

$59,60,63$

82

79

7,9

$3,8,9,33,48$, 108

76 decontamination

diagnosis

diazepam

domestic violence

$\mathbf{E}$

elderly

$72,77,83,98,99$

e-medicine

emergency health services $1,3,6,7,8,9$,

$10,12,17,21,23,35,36$,

$47,48,49,50,51,52,55$,

emergency medical services

$49,71,76$,

$92,93,95,96$

endotracheal intubation

76

epidemiology

$70,71,82,102,112$

epiglottitis

40

ethanol

etiology

etomidate

evaluation

103, 115

105

19

114,115

$$
\mathbf{F}-\mathbf{G}-\mathbf{H}
$$

fast track

geriatrics

head injury

head trauma

helmets

$$
\mathbf{I}-\mathbf{J}-\mathbf{K}
$$

influenza

information systems

injection drug use

injuries

injury surveillance

INR

interobserver agreement

intoxication

$\mathbf{L}$

legislation

lifelong learning

lorazepam

lumbar puncture

M

males

medical education informed consent

medical administration
93,97

$26,29,106$

99

29

100

61

92

39

107, 108

30

79

84,111

113

$$
\begin{aligned}
& 29 \\
& 87
\end{aligned}
$$

$31,34,46,89$

110

$59,60,65,66$, $67,68,69,74$ $16,18,42,41$, $86,87,88,90$ mental health

methanol

methicillin resistance

methodology

midazolam

minor head injury

myocardial infarction

112

114

39

$11,12,13,14,15$

19

84,106

21,23

$\mathbf{N}-\mathbf{O}$

nurse practitioner 97

occupational health 95

osmolar gap 114

Ottawa Ankle Rules 45

outcomes

$61,63,69$

overcrowding

pain assessment $\mathbf{Q}$

pain management

107

patient education

patient satisfaction

pediatrics

$2,53,54,55$

$56,57,81,91$

peer review

pelvic fracture

83

pharmacy services $\quad 74$

pharyngitis 78

physician evaluation $\quad 59$

physician satisfaction $\quad 73$

pneumonia 100

practice guidelines $\quad 79$

prediction tools 93

prehospital 91, 107

prevention 105, 108

procedural sedation $\quad 19,73,89$

procedures $\quad 16,96$

propofol

public health

quality improvement

$10,37,62$

$58,64,65$,

$67,68,69,79$,

$\mathbf{R}$

randomized controlled trials

research

13

research grant

$11,13,15,57$

research methodology

resuscitation

resuscitation termination

$44,47,50,51,55$

retrospective study

risk factors $\quad 104$

rural medicine 
$\mathbf{S}$

self-injury

silver sulfadiazine

spinal cord injuries

sports medicine

steroids

subarachnoid hemorrhage

substance abuse

suicide

survey

systematic reviews
$\mathbf{T}$

telehealth

27 therapy

30, 105 toxicology

107 trauma

24,25

$31,34,46$

103

trauma, procedures

traumatic brain injury

triage

$3,14,33,48,61,64,109,111$

triage tools

troponin

109,111

11,15

22

$$
\mathbf{U}-\mathbf{V}-\mathbf{W}
$$

ultrasound

undergraduate training

utilization

$26,30,49,52,93$, vaccination

$96,102,103,104, \quad$ vancomycin

96 vertebral fractures

whiplash

workforce
$7,35,36$

100

39

105

28 